\title{
Mental Health and Obesity: A Meta-Analysis
}

\author{
Alejandro Magallares • Jose Luis Pais-Ribeiro
}

Received: 17 September 2012 / Accepted: 7 March 2013

(C) Springer Science+Business Media Dordrecht and The International Society for Quality-of-Life Studies (ISQOLS) 2013

\begin{abstract}
The relationship between obesity and psychological distress is not clear because research conducted so far is not conclusive, with some studies finding that obese people report less mental health than normal-weight individuals, whilst others find that obesity may work as a protective factor that prevents people with weight problems from developing mental health issues. In this meta-analysis we review research that compares the mental health (measured with SF-36) of class I obese people (Body Mass Index between 30 and 34.99) with normal weight people (Body Mass Index between 18 and 24.99) in non-clinical adult populations. The metaanalysis conducted assumed a random-effects model and a weighted mean effect size was calculated $(d)$, together with its statistical significance and confidence interval. Results reveal that obese women report less mental health than normal weight females $(d=-.26)$ but that obese men show more mental health than normal weight individuals $(d=.62)$. The results give support to the so-called "Jolly Fat" hypothesis.
\end{abstract}

Keywords Obesity · Mental health · SF-36 · "Jolly Fat" hypothesis

The association between obesity in adults and mental health has been an issue of controversy for decades and the nature of the relationship between excessive weight and psychological distress continues to be debated by researchers and clinicians alike.

\footnotetext{
A. Magallares

School of Psychology, Social Psychology Department, Universidad Nacional de Educacion a Distancia (UNED), Madrid, Spain

J. L. Pais-Ribeiro

School of Psychology, Universidade de Porto, Porto, Portugal

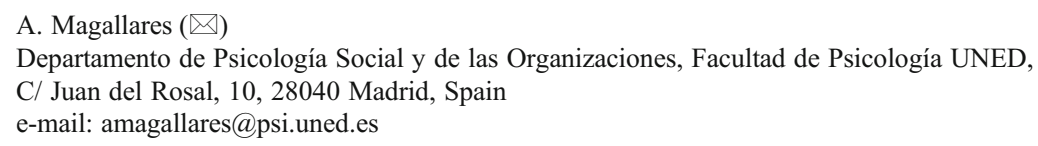


While several studies suggest that obesity increases the risk of suffering poorer mental health (see for example, Friedman and Brownell 1995), it has also been reported that obese people are likely to have higher levels of mental health (Crisp and McGuiness 1976), the so-called "Jolly Fat" hypothesis (see more recently, Han et al. 2009).

Many studies support the idea that obesity has an important role in the deterioration of health-related quality of life (in both physical and mental health domains), as recent works have shown (Atlantis et al. 2012), but there are also researchers that claim that the effects of obesity on mental health are unclear (Roberts et al. 2002). The supporters of this last hypothesis ("Jolly fat") posit that although a substantial number of the overweight and obese report less quality of life than normal weight individuals, excess weight and obesity are not necessarily depressive (Jansen et al. 2008). According to these authors, men are less vulnerable than women to the negative effect of weight on mental health (Garner et al. 2012a, b) and there is evidence that supports the idea that obese men report more quality of life than normal weight individuals in adult populations (Costa-Font and Gil 2006). In other words, there is evidence that obesity is prospectively associated with low quality of life (Faith et al. 2011) but, at the same time, there are also studies that show a positive association between Body Mass Index (BMI) and psychological distress in women (Yu et al. 2011).

As these last results may suggest, these contradictory outcomes may be explained by sex differences. The reviewed literature suggests that men may suffer less psychological distress than women, but some of the studies include in their analysis both men and women. For this reason, we have conducted a meta-analysis in order to see if there is a real connection between mental health and obesity. This relationship will be tested for both men and women, to see if there are significant differences, taking into account the sex of the participants.

\section{Obesity}

An increasing number of people face the burden of obesity, which is defined as a BMI of $\geq 30 \mathrm{~kg} / \mathrm{m}^{2}$ (Haslam and James 2005), although the World Health Organization (WHO) international classification (WHO 1995) defines obesity at different levels (for example, BMI $\geq 40 \mathrm{~kg} / \mathrm{m}^{2}$ is called massive or morbid obesity). Obesity is an important risk factor for other morbidities, such as type II diabetes, hypertension, coronary artery disease, and has been shown to reduce life expectancy (Haslam and James 2005). Estimates of prevalence in adult populations show that there is a great variability between countries (between $1 \%$, in Vietnam, for example, and $56 \%$, in Tonga) but the rates in developed countries are especially high and alarming (International Association for the Study of Obesity, IASO, 2012). This worldwide epidemic is a concern to health professionals (King 2011), because besides obesity being closely linked to risk factors associated with impaired health, it may also have an impact on the health-related quality of life (HRQoL) as many studies suggest (see for example, Jia and Lubetkin 2007). Although epidemiologic studies have demonstrated a clear impact of obesity on the perception of physical health as measured by several instruments (especially with the Short-Form 36 or SF-36 scale) for HRQoL, there is significant controversy regarding perception of mental health. As stated previously, while some studies find a negative link between HRQoL and BMI (Zhao et al. 2011), others suggest 
that obesity can be a protective factor that prevents individuals from developing mental health problems (Palinkas et al. 1996). For this reason, a meta-analysis that may help to have a clearer picture of this topic is needed. Specifically, we will focus on the impact of obesity in mental health, measured with the SF-36 in adult populations for men and women (Callegari et al. 2005).

\section{SF-36}

One of the most straightforward ways of measuring HRQoL is through the use of health status measures where patients are asked to rate different aspects of their life (Ware and Sherbourne 1992). Perhaps the most commonly used measure in HRQoL research is the Short-Form 36 or SF-36, a generic measure to assess important HRQoL domains relevant to patients suffering from a wide range of medical conditions, such as obesity (Wee et al. 2008). The SF-36 consists of 36 items distributed by eight HRQoL domains (physical functioning, role limitations due to physical problems, bodily pain, general health, vitality, social functioning, role limitations due to emotional problems, and mental health) that comprise two summary measures: the physical component summary (the first four domains) and the mental component summary (the last four domains). One of the major advantages of using the SF-36 in studies of obesity is that it allows for HRQoL scores to be compared to scores in people with normal weight (Ware et al. 2000).

The mental health component (the aggregation of the scores in the subscales of vitality, social functioning, role limitations due to emotional problems, and mental health) has been used in the obesity field to measure how people with weight problems are affected by their illness compared with normal weight individuals (see a recent example in Knoesen et al. 2012).

Additionally, it is important to note that the SF-36 mental health component measures the occurrence and extent of psychological distress (usually anxiety and depression related distress states) (Ware et al. 1993). Research shows that the mental health domain (one of the four domains that constitute the mental health component) is a good tool to screen depression (Yamazaki et al. 2005). For these reasons, studies conducted with the SF-36 will be taken into account to conduct the meta-analysis.

The aim of this meta-analysis is to provide information about the relationship between HRQoL (measured with the mental health component of SF-36) and obesity in adult populations because, as we have demonstrated, there is no consensus in the scientific community about the link between BMI and mental health in obese individuals compared with normal weight participants. These differences may be explained because not all authors have included moderator variables that may partially explain the results (such as sex). Therefore, it is the goal of this investigation to determine if there are differences between men and women, since the existing literature indicates that women are at highest risk of suffering poorer mental health than obese male. Furthermore, based on the reviewed literature, it is expected than class I obese men will report more quality of life (measured with the mental health component of the SF-36) than male individuals with normal weight, and that class I obese women will show less well-being (measured with the mental health component of the SF-36) than female participants without weight problems. 


\section{Materials and Methods}

\section{Selection of Studies}

This meta-analysis comprises empirical studies in the English, Spanish, Italian or Portuguese scientific literature (languages spoken by the research team). Included were studies with non-clinical samples of adult, but not elderly or child, populations, who were normal weight (BMI between 18 and 24.99) or class I obese (BMI between 30 and 34.99) (WHO 2000).

We excluded from our analysis morbid obesity populations (Lerdal et al. 2011), child obesity (Johnson et al. 2012), and erderly populations (Kuo et al. 2011), to focus on how class I obesity may have an effect on mental health in adult populations.

The decision to select non-clinical samples and focus on healthy individuals, was aimed at avoiding the possible incidence of physical health and chronic diseases in the results, due to their proven relationship with mental health (van der Windt et al. 2008), and because recently it has been showed that healthy obese individuals may not suffer psychological distress (Hamer et al. 2012).

Non-published works were included (dissertations and conference papers) in order to avoid the publication bias (Rothstein et al. 2005; Song et al. 2010).

We limited our search to the frequently used SF-36 questionnaires, because it was our goal to compare obese populations with normal weight samples. For this reason, studies with instruments developed to measure just HRQoL for obese populations, such as the Impact of Weight on Quality of Life-Life Questionnaire (Kolotkin et al. 2001; Kolotkin and Crosby 2002), were not selected.

The search strategy for identification of relevant literature was carried out in the following way. PubMed, MEDLINE, PsycArticles, Fonte Academica, Academic Search Complete, MedicLatina, and PsycINFO databases were systematically searched for the periods 1960 until 2012 with the following key words (mental health) AND (SF-36) AND (obesity), which yielded 127 titles. After the exclusion of studies with children, the elderly, morbid obesity and clinical groups, as well as non-empirical studies, 20 titles resulted. The abstracts were evaluated to determine whether the article was about HRQoL as it related to normal groups and obese groups. Only studies that applied the generic SF-36 questionnaire (12 articles) were selected. The studies selected can be seen in Table 1 .

The following data were extracted from the selected studies: BMI, age, sex, and means and standard deviations of the HRQoL variable (the mental health component of the SF-36) in the control group (normal weight individuals), and obese group, for both men and women.

\section{Materials}

As mentioned before, studies that measured the mental health component of the SF-36 in non-clinical adult populations, comparing class I obese and normal weight individuals were selected.

The SF-36 has adequate psychometric characteristics, including good construct validity, high internal consistency, and high test-retest stability, according to the authors that originally developed the instrument (Ware and Sherbourne 1992). 
Table 1 Studies selected to conduct the meta-analysis (by year of publication)

\begin{tabular}{lll}
\hline Authors & Place & $\begin{array}{l}\text { Age of } \\
\text { participants (years) }\end{array}$ \\
\hline Burns et al. (2001) & Bilthoven (Netherlands) & $20-59$ \\
Larsson et al. (2002) & Goteborg(Sweden) & $35-64$ \\
Surtees et al. (2004) & Norfolk (United Kingdom) & $41-80$ \\
Tsai et al. (2004) & Taiwan (China) & $35-61$ \\
Hopman et al. (2007) & Montreal (Canada) & $25-$ \\
Saraç et al. (2007) & Izmir (Turkey) & $20-65$ \\
Santos and Pereira (2008) & Lisboa (Portugal) & $28-56$ \\
Vasiljevic et al. (2008) & Belgrade (Serbia) & $18-$ \\
Renzaho et al. (2010) & Deakin University (Australia) & $21-64$ \\
Bentley et al. (2011) & Health Analytic Research & $35-89$ \\
Wang et al. (2011) & (United States of America) & $18-80$ \\
Franco et al. (2012) & Shanghai (China) & $18-$ \\
\hline
\end{tabular}

Scores range from 0 (poor mental health) to 100 (good mental health). Higher scores on the mental health component of the SF-36 reflect greater quality of life. According to the original authors, a population difference of $\geq 5$ points on this scale is considered clinically significant (Ware and Sherbourne 1992).

\section{Computation of the Effect Size Index}

The effect size index was used (Lipsey and Wilson 2001; Sánchez-Meca and Botella 2010). The standardized mean difference, $d$, is defined as the difference between the obese group and the control group mean, divided by the square of the standard deviation in the obese group and the control group divided by 2, weighting the different samples sizes (Cooper 2010; Sánchez-Meca and Marin-Martinez 1998). A positive $d$ index means that the mean in the mental health component of the SF-36 in the obese group was better than that in the control group (normal weight). The effect size calculations were subjected to reliability analysis. They produced, on average, an intraclass correlation coefficient (ICC) for continuous ratings and two evaluators, calculated with SPSS of .95 (Pearson's $r$ ) between the estimates obtained by the authors of the study.

The results for each study were classified as a function of the sex. Therefore, a $d$ index was calculated separately for men and women. Additionally, a $d$ index was calculated with all the participants of the studies included in the meta-analysis.

In each meta-analysis and assuming a random-effects model, a weighted mean effect size was calculated, together with its statistical significance and confidence interval. Random effects models were selected because we were not making inferences about the effect parameters in the studies that have been observed (fixed effects), but rather making inferences about the distribution of effect parameters in a population of studies, from a random sample of studies (Borenstein et al. 2010; 
Hedges and Vevea 1998). As per Cohen (1992), the effect sizes of .20, .50, and .80 were interpreted as representing an effect of small, medium, and large magnitude, respectively. According to Ferguson (2009), the effects sizes calculated for group differences should be interpreted in an alternative way [.41 (small), 1.15 (medium) and 2.70 (large)].

The next step in the statistical analysis consisted of applying the heterogeneity $C h i^{2}$ statistic and the $I^{2}$ index (which describes the percentage of total variation across studies that is due to heterogeneity rather than chance) in order to examine if the individual effect sizes were homogeneous around the mean effect size (Cooper et al. 2009). According to Higgins et al. (2003), values of $25 \%, 50 \%$, and $75 \%$ are considered low, moderate, and high levels of heterogeneity, respectively.

Additionally, forest plots were created in order to present the results of the metaanalysis (Anzures and Higgins 2010). Forest plots are designed to illustrate the relative strength of treatment effects in multiple quantitative scientific studies addressing the same question.

All the statistical analyses were conducted with the RevMan software (2011).

\section{Results}

Firstly, we carried out a meta-analysis for all the studies selected. The weighted mean effect size obtained for the global outcome measure in the 24 studies (12 for men and 12 for women: 30668 participants) was $d=.12[.1, .15]$ and statistically significant $(Z=11.13 ; p<.01)$. Using Cohen (1992) and Ferguson (2009), we can consider this mean effect size to be of small magnitude. This result means that obese people, without considering sex as a moderator variable, report better mental health than normal weight individuals do. The heterogeneity of the sample was measured with a $\mathrm{Chi}^{2}$ (8330.64, degrees of freedom $=23 ; p<.01 ; I^{2}=99 \%$ ). According to the Higgins et al. (2003) criterion, the heterogeneity of the effect size found is high.

Secondly, we conducted separate meta-analyses for women and men. Table 2 shows the different sizes of the samples for both women and men.

The female obese group was composed of 7321 individuals and the control group (normal weight individuals) included 16373 women participants. On the other hand, the male obese group was composed of 4845 individuals and the control group was 10129 male participants.

\section{Women}

The weighted mean effect size obtained for the global outcome measure in the 12 studies (see Table 3 and Fig. 1) was $d=-.26[-.27,-.21]$ and statistically significant $(Z=16.44 ; p<.01)$. Following Cohen (1992) and Ferguson (2009), we can consider this mean effect size to be of small magnitude. This result means that obese women suffer poorer mental health compared to the normal weight female group.

The heterogeneity of the sample was measured with a $\mathrm{Chi}^{2}$ (4856.04, degrees of freedom $=11 ; p<.01 ; I^{2}=99 \%$ ). According to the Higgins et al. (2003) criterion, the heterogeneity of the effect sizes found is high.

A population difference of $\geq 5$ points on the SF- 36 is considered clinically significant (Ware and Sherbourne 1992), which means that the only study with differences 
Table 2 Samples sizes of the different studies (by year of publication)

\begin{tabular}{lllll}
\hline Studies & $\begin{array}{l}N \text { (control } \\
\text { group women) }\end{array}$ & $\begin{array}{l}N \text { (obese } \\
\text { group women) }\end{array}$ & $\begin{array}{l}N \text { (control } \\
\text { group men) }\end{array}$ & $\begin{array}{l}N \text { (obese } \\
\text { group men) }\end{array}$ \\
\hline Burns et al. (2001) & 1176 & 1176 & 1021 & 1021 \\
Larsson et al. (2002) & 1070 & 136 & 698 & 137 \\
Surtees et al. (2004) & 4276 & 1155 & 2489 & 856 \\
Tsai et al. (2004) & 1327 & 175 & 1033 & 201 \\
Hopman et al. (2007) & 2367 & 1035 & 843 & 444 \\
Saraç et al. (2007) & 250 & 1498 & 150 & 254 \\
Santos and Pereira (2008) & 58 & 58 & 38 & 38 \\
Vasiljevic et al. (2008) & 1168 & 121 & 526 & 100 \\
Renzaho et al. (2010) & 2114 & 689 & 1476 & 793 \\
Bentley et al. (2011) & 733 & 691 & 421 & 456 \\
Wang et al. (2011) & 1138 & 56 & 1038 & 49 \\
Franco et al. (2012) & 696 & 531 & 396 & 496 \\
\hline
\end{tabular}

between the obese and the control group is the one by Saraç et al. (2007), although there are other studies that have shown high effect sizes. When this study was removed, the effect size remained the same $(d=-.26)$.

\section{Men}

The weighted mean effect size obtained for the global outcome measure in the 12 studies (see Table 4 and Fig. 2) was $d=.62[.59, .65]$ and statistically significant $(Z=36.31 ; p<.01)$. Following Cohen (1992), we can consider this mean effect size to be of medium magnitude. According to the Ferguson (2009) criterion it would be small. This result means that obese men have better mental health compared to the normal weight male group.

Table 3 Effect sizes for women (by size of $d$ )

\begin{tabular}{ll}
\hline Study & $d$ [Confidence interval] \\
\hline Saraç et al. (2007) & $-15.80[-17.66,-13.94]$ \\
Larsson et al. (2002) & $-2.80[-3.15,-2.45]$ \\
Burns et al. (2001) & $-1.80[-1.86,-1.74]$ \\
Vasiljevic et al. (2008) & $-1.70[-5.44,2.04]$ \\
Bentley et al. (2011) & $-1.50[-1.72,-1.28]$ \\
Santos and Pereira (2008) & $-1.39[-5.50,2.72]$ \\
Wang et al. (2011) & $-0.66[-3.08,1.76]$ \\
Hopman et al. (2007) & $-0.10[-0.18,-0.02]$ \\
Renzaho et al.(2010) & $0.00[-0.85,0.85]$ \\
Tsai et al. (2004) & $0.20[0.09,0.31]$ \\
Surtees et al. (2004) & $0.40[-0.23,1.03]$ \\
Franco et al. (2012) & $0.50[0.46,0.54]$ \\
\hline
\end{tabular}


Fig. 1 Forest plot in women

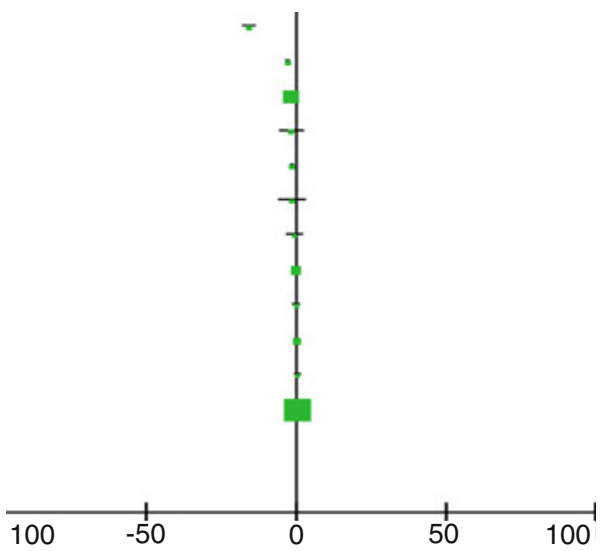

The heterogeneity of the sample was measured with a $C h i^{2}$ (2010.17, degrees of freedom $=11 ; p<.01 ; I^{2}=99 \%$ ). According to the Higgins et al. (2003) criterion, the heterogeneity of the effect sizes found is high.

A population difference of $\geq 5$ points on the SF-36 is considered clinically significant (Ware and Sherbourne 1992), which means that the only study with differences between the obese and the control group is the one made by Saraç et al. (2007), although there are other studies that have shown high effect sizes. When this study was removed, the effect size remained the same $(d=.62)$.

\section{Discussion}

The results of the meta-analysis reveal that when sex is not taken into account, obese individuals report more quality of life $(d=.12)$ than normal weight people, although the found effect is small (according to Cohen 1992, and Ferguson 2009). Wadden and Stunkard (1987) observe that obesity is associated with psychological distress in

Table 4 Effect sizes for men (by size of $d$ )

\begin{tabular}{ll}
\hline Study & $d$ [Confidence interval] \\
\hline Saraç et al. (2007) & $-14.50[-16.93,-12.07]$ \\
Hopman et al. (2007) & $-2.20[-2.61,-1.79]$ \\
Vasiljevic et al. (2008) & $-1.10[-4.86,2.66]$ \\
Tsai et al. (2004) & $-0.10[-0.19,-0.01]$ \\
Santos and Pereira (2008) & $-0.07[-3.96,3.82]$ \\
Surtees et al. (2004) & $0.00[-0.70,0.70]$ \\
Larsson et al. (2002) & $0.10[-0.05,0.25]$ \\
Franco et al. (2012) & $0.20[0.15,0.25]$ \\
Renzaho et al. (2010) & $0.30[-0.49,1.09]$ \\
Bentley et al. (2011) & $1.20[0.91,1.49]$ \\
Burns et al. (2001) & $1.60[1.54,1.66]$ \\
Wang et al. (2011) & $2.21[0.20,4.22]$
\end{tabular}


Fig. 2 Forest plot in men

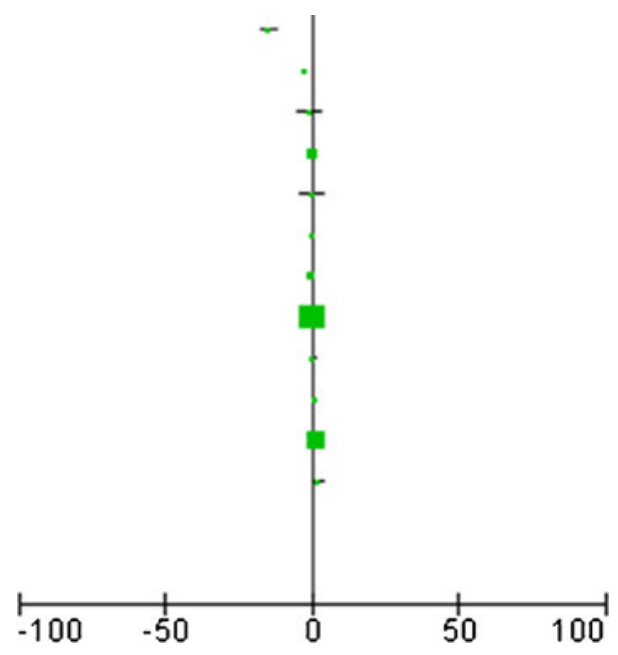

some obese persons, particularly those that have accompanying chronic conditions. Some papers have found that obese patients with chronic conditions are more likely to be particularly vulnerable to suffering psychological distress (Doll et al. 2000). Because of this relationship between health and psychological distress, studies selected were of non-clinical populations, in order to avoid the possible influence of chronic diseases in the results (van der Windt et al. 2008). When non-clinical samples are selected, the results show that class I obese individuals do not report less quality of life than normal weight individuals do, as this meta-analysis has also demonstrated.

Additionally, the result of the meta-analysis suggests that the relationship between BMI and HRQoL differs between men and women (Garner et al. 2012a, b). Our study found an inverse relationship between obesity and psychological distress symptoms in men $(d=.62)$, but not in women $(d=-.26)$. These results suggest that obesity may have a beneficial effect on mental health for men but not for women. This inverse association between obesity and mental health could be considered as supporting the "Jolly Fat" hypothesis (Crisp and McGuiness 1976). This hypothesis states that men that are overweight suffer less psychological distress symptoms than men with normal weight (Kivimaki et al. 2011), and recently several studies have shown that this pattern can be found in older people (Kim et al. 2010) and adolescents (Revah-Levy et al. 2011) as well.

Other meta-analysis conducted with other quality of life measures also reports similar results regarding sex differences in the obese field. For example, a significant positive association in depression was found for females, and a smaller nonsignificant association for males (de Wit et al. 2010). In the case of self-esteem, the overall mean effect size found was moderate, with lower self-esteem associated with heavier weight, and the effect sizes were larger for women than for men (Miller and Downey 1999).

This result, in which obese women report less mental health than normal weight women, and less than men, can be interpreted as a consequence of the fact that women generally report greater reductions in mental health when compared to men. 
According to the reviewed literature, there are significant differences in the types of mental illness affecting women and men (the most common mental illnesses experienced by women are depression, bipolar disorder and anxiety), so this meta-analysis would be another manifestation of this general effect (Astbury 2001; Patel 2005; WHO 2001).

Additionally, it is important to point out that the differences between men and women may be explained because Westem sociocultural norms emphasize and idealize extreme slimness for women but not for men (Murnen 2011). Thinness internalization is fostered by perceived pressure to be slim which is more important in women than in men (Juarez et al. 2012), and since men are less likely to internalize the slimness ideal, they report more mental health, because they receive fewer pressuring messages in interactions with family and friends (see for example, Thompson et al. 1999).

The current SF-36 findings suggest that obese women experience limitations in their daily life that are not fully explained by the magnitude of excess weight (the effect size found is low). An implication of this finding is that weight reduction alone (trough bariatric surgery or hypo caloric diet and exercise) will not suffice when attempting to positively affect the mental health of these individuals. Research has shown, for example, that obese people have to face strong social rejection and exclusion in several areas because of their weight (Myers and Rothblum 2010), as demonstrated by a number of studies that suggest anti-fat attitudes are widespread (Puhl and Heuer 2009). For this reason, we believe that studies should include stigma measures in order to achieve a better understanding of mental health in obese individuals. According to Carr and Friedman (2005), the contradictory results, reviewed in the introduction section, may be explained if some moderating psychosocial variables (such as discrimination) are taken into account. Given the heterogeneity of the results found for both men and women (according to the $I^{2}$ indexes) we believe it would be necessary to increase understanding of the relationships between BMI and psychological distress, and future investigations in the mental health field for obese individuals should bear in mind these type of variables.

The current study is subject to some limitations that deserve mention. First, it is important to take into account the design of the studies. Typically, cross-sectional approaches have been used (all the studies used to conduct the meta-analysis are of this nature). However, recently, longitudinal studies have been conducted (see for example, Cameron et al. 2012), which suggests that there is a bidirectional relationship between obesity and mental health: obese individuals report more psychological distress, and at the same time, people with low levels of well-being are at highest risk to develop weight problems. Unfortunately, there are not many longitudinal studies that have controlled both effects. For this reason, we believe that future studies about the relationship between BMI and quality of life in obese people should be longitudinal. Second, the $d$ effect sizes used in this meta-analysis are based on an artificial categorization of the BMI variable reported by the original studies included. We believe that this dichotomization (between class I obesity and normal weight individuals) leads to losses of statistical power that may be affecting the results. Despite these limitations, the study provides useful data about mental health and obesity.

To summarize, research on obesity has tended to focus on the mental health of obese people, on the assumption that it should be lower than that of normal weight people (Friedman and Brownell 1995). For this reason, an important number of 
researchers have examined the relationship between obesity (as measured by BMI) and HRQoL, using generic instruments like the SF-36 (see for example, Kolotkin, Meter and Williams 2001). However, studies conducted to date are not conclusive, because some works support the so-called "Jolly Fat" hypothesis (Crisp et al. 1980). This meta-analysis has found that only women suffer less mental health than normal weight individuals, however this effect is low, which means that there may be other variables related with the psychological distress in obese female that explain this result (see for example, Puhl et al. 2010). In the case of men, it has been shown that class I obese individuals may have better mental health compared with people with normal BMIs, which gives support to the idea that obesity may work as a protective factor against psychological distress (Crisp and McGuiness 1976), and even physical health (Dorner and Rieder 2010).

\section{References}

Anzures, J., \& Higgins, J. (2010). Graphical displays for meta-analysis: an overview with suggestions for practice. Research Synthesis Methods, 1, 66-80. doi:10.1002/jrsm.6.

Astbury, J. (2001). Gender disparities in mental health. World Health Organisation Round Tables.

Atlantis, E., Goldney, R., Eckert, K., \& Taylor, A. (2012). Trends in health-related quality of life and health service use associated with body mass index and comorbid major depression in South Australia, 19982008. Quality of Life Research, 21, 1695-1704. doi:10.1007/s11136-011-0101-7.

*Bentley, T., Palta, M., Paulsen, A., Cherepanov, D., Dunham, N., Feeny, D., et al. (2011). Race and gender associations between obesity and nine health-related quality-of-life measures. Quality of Life Research, 20, 665-674. doi:10.1007/s11136-011-9878-7.

Borenstein, M., Hedges, L., Higgins, J., \& Rothstein. (2010). A basic introduction to fixed-effect and random-effects models for meta-analysis. Research Synthesis Methods, 1, 97-111. doi:10.1002/ jrsm.12.

*Burns, C., Tijhuis, M., \& Seidell, J. (2001). The relationship between quality of life and perceived body weight and dieting history in Dutch men and women. International Journal of Obesity, 25, 1386-1392.

Callegari, A., Michelini, I., Sguazzin, C., Catona, A., \& Klersy, C. (2005). Efficacy of the SF-36 questionnaire in identifying obese patients with psychological discomfort. Obesity Surgery, 15, 254 260. doi:10.1381/0960892053268255.

Cameron, A., Magliano, D., Dunstan, D., Zimmet, P., Hesketh, K., \& Shaw, J. (2012). A bi-directional relationship between obesity and health-related quality of life: evidence from the longitudinal AusDiab study. International Journal of Obesity 36, 295-303. doi:10.1038/ijo.2011.103.

Carr, D., \& Friedman, M. A. (2005). Is obesity stigmatizing? Body weight, perceived discrimination, and psychological well-being in the United States. Journal of Health and Social Behavior, 46, 244-259.

Cohen, J. (1992). A power primer. Psychological Bulletin, 112, 155-159.

Cooper, H. (2010). Research synthesis and meta-analysis: A step by step approach. Thousand Oaks: Sage.

Cooper, H., Hedges, L. V., \& Valentine, J. C. (Eds.). (2009). The handbook of research synthesis and metaanalysis (2nd ed.). New York: Russell Sage.

Costa-Font, J., \& Gil, J. (2006). Revisiting the 'Fat and Jolly' hypothesis: socio-environmental determinants of obesity and depression in Spain. Socio-Economic Review, 4, 513-542. doi:10.1093/ser/mwl018.

Crisp, A. H., \& McGuiness, B. (1976). Jolly fat: relation between obesity and psychoneurosis in general population. British Medicine Journal, 1, 7-9.

Crisp, A. H., Queenan, M., Sittampaln, Y., \& Harris, G. (1980). 'Jolly fat' revisited. Journal of Psychosomatic Research, 24, 233-241.

de Wit, L., Luppino, F., van Straten, A., Penninx, B., Zitman, F., \& Cuijpers, P. (2010). Depression and obesity: a meta-analysis of community-based studies. Psychiatry Research, 178, 230-235. doi:10.1016/j.psychres.2009.04.015.

Doll, H., Petersen, S., \& Stewart-Brown, S. (2000). Obesity and physical and emotional well-being: associations between body mass index, chronic illness, and the physical and mental components of the SF-36 questionnaire. Obesity Research, 8, 160-170. doi:10.1038/oby.2000.17. 
Dorner, T. E., \& Rieder, A. (2010). Obesity paradox or reverse epidemiology: is high body weight a protective factor for various chronic conditions. Deutsche Medizinische Wochenschrift, 135, 413-418.

Faith, M., Butryn, M., Wadden, T., Fabricatore, A., Nguyen, A., \& Heymsfield, S. (2011). Evidence for prospective associations amongdepressionandobesity in population-based studies. Obesity Reviews, 12, 438-453. doi:10.1111/j.1467-789X.2010.00843.x.

Ferguson, C. (2009). An effect size primer: a guide for clinicians and researchers. Professional Psychology: Research \& Practice, 40, 532-538. doi:10.1037/a0015808.

*Franco, O., Wong, Y., Kandala, N., Ferrie, J., Dorn, J., Kivimaki, M., et al. (2012). Cross-cultural comparison of correlates of quality of life and health status: the Whitehall II Study (UK) and the Western New York Health Study (US). European Journal of Epidemiology, 27, 255-265. doi:10.1007/ s10654-012-9664-Z

Friedman, M., \& Brownell, K. (1995). Psychological correlates of obesity: moving to the next research generation. Psychological Bulletin, 117, 3-20.

Garner, R., Feeny, D., Thompson, A., Bernier, J., McFarland, B., Huguet, N., et al. (2012a). Bodyweight, gender, and quality of life: a population-based longitudinal study. Quality of Life Research, 21, 813825. doi:10.1007/s11136-011-9989-1.

Garner, R., Feeny, D., Thompson, A., Bernier, J., McFarland, B., ... Blanchard, C. (2012b). Bodyweight, gender, and quality of life: a population-based longitudinal study. Quality ofLife Research, 21, 813825. doi:10.1007/s11136-011-9989-1

Han, C., Jo, S. A., Seo, J. A., Kim, B. G., Kim, N. H., Jo, I., et al. (2009). Adiposity parameters and cognitive function in the elderly: application of "Jolly Fat" hypothesis to cognition. Archives of Gerontology and Geriatrics, 49, 133-138.

Hamer, M., Batty, G., \& Kivimaki, M. (2012). Risk of future depression in people who are obese but metabolically healthy: the English longitudinal study of ageing. Molecular Psychiatry, 17, 940-945. doi:10.1038/mp.2012.30.

Haslam, D. W., \& James, W. P. (2005). Obesity. Lancet, 366, 1197-1209. doi:10.1016/S0140-6736(05)67483-1.

Hedges, L. V., \& Vevea, J. L. (1998). Fixed and random-effects models in meta-analysis. Psychological Methods, 3, 486-504.

Higgins, J., Thompson, S., Deeks, J., \& Altman, D. (2003). Measuring inconsistency in meta-analyses. British Medicine Journal, 327, 557-560. doi:10.1136/bmj.327.7414.557.

*Hopman, W., Berger, C., Lawrence, J., Barr, S., Gao, Y., Prior, J., et al. (2007). The association between body mass index and health-related quality of life: data from CaMos, a stratified population study. Quality of Life Research, 16, 1595-1603. doi:10.1007/s11136-007-9273-6

International Association for the Study of Obesity. (2012). Global prevalence of adult population. London: IASO.

Jansen, A., Havermans, R., Nederkoorn, C., \& Roefs, A. (2008). Jolly fat or sad fat?: subtyping non-eating disordered overweight and obesity along an affect dimension. Appetite, 51, 635-640. doi:10.1016/ j.appet.2008.05.055.

Jia, H., \& Lubetkin, E. I. (2007). The impact of obesity on health-related quality-of-life in the general adult US population. Journal of Public Health, 27, 156-64. doi:10.1093/pubmed/fdi025.

Johnson, B., Kremer, P., Swinburn, B., \& de Silva-Sanigorski, A. (2012). Multilevel analysis of the Be Active Eat Well intervention: environmental and behavioural influences on reductions in child obesity risk. International Journal of Obesity, 36, 901-907. doi:10.1038/ijo.2012.23.

Juarez, L., Soto, E., \& Pritchard, M. (2012). Drive for muscularity and drive for thinness: the impact of proanorexia websites. Eating Disorders, 20, 99-112. doi:10.1080/10640266.2012.653944.

Kim, E., Song, J. H., Hwang, J., Ahn, K., Kim, J., Koh, Y. H., et al. (2010). Obesity and depressive symptoms in elderly Koreans: evidence for the "Jolly Fat" hypothesis from the Ansan Geriatric (AGE) Study. Archives of Gerontology and Geriatrics, 51, 231-234. doi:10.1016/j.archger.2009. 10.014 .

King, D. (2011). The future challenge of obesity. Lancet, 378, 743-744. doi:10.1016/S0140-6736(11) 61261-0.

Kivimaki, M., Jokela, M., \& Batty, G. D. (2011). Does obesity really protect against psychological distress? Examining the 'fat-jolly' versus 'fat-sad' hypotheses using Mendelian randomization. Journal Of Internal Medicine, 269, 519-20. doi:10.1111/j.1365-2796.2011.02357.x.

Knoesen, N., Mancuso, S., Thomas, S., Komesaroff, P., Lewis, S., \& Castle, D. (2012). Relationship between severity of obesity and mental health: an Australian community survey. Asia-Pacific Psychiatry, 4, 67-75. doi:10.1111/j.1758-5872.2011.00164.x.

Kolotkin, R. L., Crosby, R. D., Kosloski, K. D., \& Williams, G. R. (2001). Development of a brief measure to assess quality of life in obesity. Obesity Research, 9, 102-111. doi:10.1038/oby.2001.13. 
Kolotkin, R. L., Meter, K., \& Williams, G. R. (2001). Quality of life and obesity. Obesity Review, 2, $219-229$. Kolotkin, R. L., \& Crosby, R. D. (2002). Psychometric evaluation of the Impact Of Weight On Quality Of Life-Lite Questionnaire (IWQOL-Lite) in a community sample. Quality of Life Research, 1, 157-171. doi:10.1023/A:1015081805439.

Kuo, S., Lin, K., Chen, C., Chuang, Y., \& Chen, W. (2011). Depression trajectories and obesityamong the elderly in Taiwan. Psychological Medicine, 41, 1665-1676. doi:10.1017/S0033291710002473.

*Larsson, U., Karlsson, J., \& Sullivan, M. (2002). Impact of overweight and obesity on health-related quality of life - a Swedish population study. International Journal of Obesity and Related Metabolic Disorders, 26, 417-424.

Lerdal, A., Andenæs, R., Bjørnsborg, E., Bonsaksen, T., Borge, L., \& Fagermoen, M. (2011). Personal factors associated with health-related quality of life in persons with morbid obesity on treatment waiting lists in Norway. Quality of Life Research, 20, 1187-1196. doi:10.1007/s11136-011-9865-z.

Lipsey, M., \& Wilson, D. (2001). Practical meta-analysis. Thousand Oaks: Sage.

Miller, C. T., \& Downey, K. T. (1999). A meta-analysis of heavyweight and self-esteem. Personality and Social Psychology Review, 3, 68-84.

Murnen, S. (2011). Gender and body images. In T. Cash \& L. Smolak (Eds.), Body image: A handbook of science, practice, and prevention (pp. 173-179). New York: Guilford Press. doi:10.1016/B978-0-12384925-0.00019-5.

Myers, A., \& Rothblum, E. (2010). Coping with prejudice and discrimination based on weight. In J. L. Chin (Ed.), The psychology of prejudice and discrimination: A revised and condensed edition (pp. 187-197). Santa Barbara: Praeger.

Palinkas, L. A., Wingard, D. L., \& Barrett-Connor, E. (1996). Depressive symptoms in overweight and obese older adults: a test of the "jolly fat" hypothesis. Journal of Psychosomatic Research, 40, 59-66. doi:10.1016/0022-3999(95)00542-0.

Patel, V. (2005). Gender in mental health research. Italy: World Health Organisation.

Puhl, R., \& Heuer, C. (2009). The stigma of obesity: a review and update. Obesity, 17, 941-964. doi:10.1038/oby.2008.636.

Puhl, R., Heuer, C., \& Brownell, K. (2010). Stigma and social consequences of obesity. In P. Kopelman, I. Caterson, \& W. Dietz (Eds.), Clinical obesity in adults and children (pp. 25-40). New York: WileyBlackwell.

*Renzaho, A., Wooden, M., \& Houng, B. (2010). Associations between body mass index and health-related quality of life among Australian adults. Quality of Life Research, 19, 515-520. doi:10.1007/s11136010-9610-z

Revah-Levy, A., Speranza, M., Barry, C., Hassler, C., Gasquet, I., ... \& Falissard, B. (2011). Association between Body Mass Index and depression: the "fat and jolly" hypothesis for adolescents girls. $B M C$ Public Health, 16, 1-7. doi:10.1186/1471-2458-11-649

RevMan: The Cochrane Collaboration's Review Manager (Vers. 5.1) (2011). The Cochrane Collaboration.

Roberts, R., Strawbridge, W., Deleger, S., \& Kaplan, G. (2002). Are the fat morejolly? Annals Of Behavioral Medicine, 24, 169-80.

Rothstein, H. R., Sutton, A. J., \& Borenstein, M. (Eds.). (2005). Publication bias in metaanalysis: Prevention, assessment, and adjustments. Chichester: Wiley.

Sánchez-Meca, J., \& Marin-Martinez, F. (1998). Weighting by inverse variance or by sample size in metaanalysis: a simulation study. Educational and Psychological Measurement, 58, 211-220. doi:10.1177/ 0013164498058002005.

Sánchez-Meca, J., \& Botella, J. (2010). Revisiones sistemáticas y meta-análisis: herramientas para la práctica profesional [Systematycal review and meta-analysis: profesional practice tools]. Papeles del Psicólogo, 31, 7-17.

*Santos, R., \& Pereira, J. (2008). O peso da obesidade: avaliação da qualidade de vida relacionada com a saúde em utentes de farmácias [Obesity weight: quality of life assesment related to health in pharmacy clients]. Qualidade de vida, 26, 25-37.

*Saraç, F., Parýldar, S., Duman, E., Saygýlý, F., Tüzün, M., \& Yýlmaz, C. (2007). Quality of life for obese women and men in Turkey. Preventing Chronic Disease, 4, 1-7.

Song, F., Parekh, S., Hooper, L., Loke, Y. K., Ryder, J., Sutton, A. J., et al. (2010). Dissemination and publication of research findings: an updated review of related biases. Health Technology Assessment, 14, 188-193. doi:10.3310/hta14080.

*Surtees, P., Wainwright, N., \& Khaw, K. (2004). Obesity, confidant support and functional health: crosssectional evidence from the EPIC-Norfolk cohort. International Journal of Obesity, 28, 748-758.

*Tsai, W., Yang, C., Lin, S., \& Fang, F. (2004). Impact of obesity on medical problems and quality of life in Taiwan. American Journal of Epidemiology, 160, 557-565. doi:10.1093/aje/kwh251. 
Thompson, J. K., Heinberg, L. J., Altabe, M. N., \& Tantleff-Dunn, S. (1999). Exacting beauty: theory, assessment and treatment of body image disturbance. Washington: American Psychological Association. doi:10.1037/10312-000.

van der Windt, D., Dunn, K., Spies-Dorgelo, M., Mallen, C., Blankenstein, A., \& Stalman, W. (2008). Impact of physical symptoms on perceived health in the community. Journal of Psychosomatic Research, 64, 265-274. doi:10.1016/j.jpsychores.2007.10.003.

*Vasiljevic, N., Ralevic, S., Marinkovic, J., Kocev, N., Maksimovic, M., ... \& Tomic, J. (2008). The assessment of health-related quality of life in relation to the body mass index value in the urban population of Belgrade. Health and Quality of Life Outcomes, 6, 1-10. doi:10.1186/1477-7525-6-106

Wadden, T. A., \& Stunkard, A. J. (1987). Psychopathology and obesity. Annual New York Academic Sciences, 499, 55-65.

*Wang, R., Wu, M., Ma, X., Zhao, Y., Yan, X., ... \& He, J. (2011). Body mass index and health-related quality of life in adults: a population based study in five cities of China. European Journal of Public Health, 1-6. doi:10.1093/eurpub/ckr080

Ware, J. E., Kosinski, M., \& Dewey, J. E. (2000). How to score version two of the SF-36® health survey. Lincoln: QualityMetric Incorporated.

Ware, J. E., \& Sherbourne, C. D. (1992). The MOS 36-item short-form health survey (SF-36). I. Conceptual framework and item selection. Medical Care, 30, 473-483.

Ware, J. E., Snow, K. K., Kosinski, M., \& Gandek, B. (1993). SF-36 health survey: Manual and interpretation guide. Boston: The Health Institute, New England Medical Center.

Wee, C., Davis, R., \& Hamel, M. (2008). Comparing the SF-12 and SF-36 health status questionnaires in patients with and without obesity. Health and Quality of Life Outcomes, 6, 1-7. doi:10.1186/14777525-6-11.

World Health Organization (1995). Physical status: The use and interpretation of anthropometry. WHO Technical Report Series no. 854. Geneva: WHO.

World Health Organization (2000). Obesity: Preventing and managing the global epidemic. WHO Technical Report Series 894. Geneva: WHO.

World Health Organisation. (2001). World Health Report 2001: Mental Health: New Understanding, New Hope. Geneva: WHO.

Yamazaki, S., Fukuhara, S., \& Green, J. (2005). Usefulness of five-item and three-item Mental Health Inventories to screen for depressive symptoms in the general population of Japan. Health and Quality of Life Outcomes, 3, 48. doi:10.1186/1477-7525-3-48.

Yu, N., Chen, C., Liu, C., Chau, Y., Chang, C., \& Chang, G. (2011). Association of body mass index and depressive symptoms in a Chinese community population: results from the Health Promotion Knowledge, Attitudes, and Performance Survey in Taiwan. Medical Journal, 34, 620-627.

Zhao, G., Ford, E., Li, C., Tsai, J., Dhingra, S., \& Balluz, L. (2011). Waist circumference, abdominal obesity, and depression among overweight and obese U.S.adults: National Health and Nutrition Examination Survey 2005-2006. BMC Psychiatry, 11, 130. doi:10.1186/1471-244X-11-130.

*Studies included in the meta-analysis 